\title{
The CME-flare relationship: Are there really two types of CMEs?
}

\author{
B. Vršnak, D. Sudar, and D. Ruždjak
}

\author{
Hvar Observatory, Faculty of Geodesy, Kačićeva 26, 10000 Zagreb, Croatia \\ e-mail: bvrsnak@geodet.geof.hr
}

Received 13 October 2004 / Accepted 7 February 2005

\begin{abstract}
We present a statistical analysis of 545 flare-associated CMEs and 104 non-flare CMEs observed in the heliocentric distance range 2-30 solar radii. We found that both data sets show quite similar characteristics, contradicting the concept of two distinct (flare/non-flare) types of CMEs. In both samples there is a significant fraction of CMEs showing a considerable acceleration or deceleration and both samples include a comparable ratio of fast and slow CMEs. We present kinematical curves of several fast non-flare CMEs moving at a constant speed or decelerating, i.e., behaving as expected for flare-associated CMEs. Analogously, we identified several slow flare-CMEs showing the acceleration peak beyond a height of 3 solar radii.

On the other hand, it is true that CMEs associated with major flares are on average faster and broader than non-flare CMEs and small-flare CMEs. There is a well-defined correlation between the CME speed and the importance of the associated flare. In this respect, the non-flare CMEs show characteristics similar to CMEs associated with flares of soft X-ray class B and C, which is indicative of a "continuum" of events rather than supporting the existence of two distinct CME classes. Furthermore, we inferred that CMEs whose source region cannot be identified with either flares or eruptive prominences are on average slowest. The results indicate that the magnetic reconnection taking place in the current sheet beneath the CME significantly influences the CME dynamics.
\end{abstract}

Key words. Sun: coronal mass ejections (CMEs) - Sun: flares - Sun: prominences - Sun: filaments magnetohydrodynamiques

\section{Introduction}

A comparison of the kinematics of 16 CMEs associated with (large) flares and 11 non-flare CMEs observed by the Skylab coronagraph led Gosling et al. (1976) to the conclusion that flare-associated CMEs are significantly faster than non-flare CMEs. Several years later, MacQueen \& Fisher (1983) analyzed six flare-associated CMEs and six non-flare CMEs associated with filament eruptions observed by the MK3 coronagraph at the Mauna Loa Solar Observatory, and noted that the former ones were faster, moving at approximately constant velocities, whereas the latter were slower, showing considerable accelerations. Combining their results with the results by Gosling et al. (1976) and Hildner (1977), MacQueen \& Fisher (1983) put forward the concept of two distinct classes of CMEs: the flare-associated ones, being accelerated impulsively at low heights, and the non-flare CMEs accelerated gradually over a large height range. Presently, the concept of two distinct CME classes is widely employed to interpret various aspects of CMEs (e.g., Sheeley et al. 1999; Andrews \& Howard 2001; Low \& Zhang 2002; Moon et al. 2002; Chen \& Krall 2003).

While it is true that CMEs accompanied by major flares are usually very bright, fast and wide, the concept of two distinct classes of CMEs faces serious problems when confronted with observations. The first one appears in statistical analyses: for example, Fig. 12 of Moon et al. (2002) indicates that there is a correlation between the speed of CMEs and the time-integrated soft X-ray flux of the associated flares. Similarly, Fig. 6 of Burkepile et al. (2004) reveals a distinct correlation between the kinetic energy of CMEs and the SXR peak flux in a sample of $24 \mathrm{CME} /$ flare limb-events. Finally, Moon et al. (2003) found a very good correlation between CME velocities and the associated SXR peak fluxes in two carefully selected sets of CME/flare events. The graphs show a "continuum" of events (no distinct grouping), and furthermore, a considerable number of events is slow (much slower than usually considered as typical for the flare-associated CMEs). In this respect it should be noted that the samples used in the analyzes by Gosling et al. (1976), Hildner (1977), and MacQueen \& Fisher (1983) are in fact not statistically reliable - the samples were small, preferably comprising large events. Finally, we emphasize that there is a certain controversy regarding the fraction of CMEs having "constant speed" or "constant acceleration" (for a discussion, see Sect. 5 of Andrews \& Howard 2001). The controversy arises from the fact that the judgement on the type of kinematical curve is often highly subjective, and furthermore it depends on the performance of the instruments, the measurement technique, and the morphological characteristics of a CME

The second problem is the various case-study counterexamples in the literature. For example, the two flare-CMEs studied by Zhang et al. (2004) attained the acceleration maximum at 
$R \approx 2$ and $R \approx 5$ solar radii. Similarly, Moon et al. (2004) reported three flare-associated CMEs, two of which clearly showed the acceleration phase in the range $R>2$. On the other hand, the quiescent filament (fast) eruption of 18 August 1980 showed the acceleration peak at a height of around $H=0.5$ solar radii above the solar surface (Illing \& Hundhausen 1986; Vršnak et al. 1993, and references therein).

Furthermore, the rapid acceleration stage of CMEs is closely synchronized with the rising phase of the associated soft X-ray bursts (Zhang et al. 2001; see also Vršnak et al. 2004a, and references therein). So, the duration of the CME acceleration depends on the impulsive/gradual character of the flare: CMEs accompanied by gradual flares should accelerate over a large distance range, contradicting the basic presumption of the two-class CME scheme.

In this paper we check the statistical aspect of the twoclass CME concept, by comparing a large sample of flareassociated CMEs (hereafter F-CMEs) with a sample of nonflare CMEs whose source region is revealed by disappearing filaments (hereafter DSF-CMEs). After describing the data set and the sampling procedure in Sects. 2 and 3, we present in Sects. 4 and 5 the comparison of the two samples. The results indicate that the two-class scheme has to be reformulated since there is no distinct difference between DSF-CMEs and the majority of F-CMEs. Only CMEs associated with major flares show on average larger velocities. However, even this category of CMEs involves a significant fraction of slow events. On the other hand, the sample of DSF-CMEs contains a considerable number of fast events. The outcome is interpreted and discussed in Sect. 6, emphasizing the role of magnetic field reconnection.

\section{Data}

The analysis is based on three data sets - the CME, the flare, and the disappearing filament data set. The initial CME sample consists of more than 5000 events observed in the range 2-30 solar radii by the Large Angle and Spectrometric Coronagraph (LASCO; Brueckner et al. 1995) in the period 1996-2001. The solar soft X-ray (SXR) flux measurements provided by the Geostationary Operational Enviromental Satellites (GOES; Donelly \& Unzicker 1974), recorded in the same period 11693 distinct flare events in the 1-8 Å GOES channel. The sample of DSF-CMEs is selected utilizing the disappearing filament data reported in the Solar Geophysical Data catalogues by various ground-based patrol observatories.

The analysis of the CME kinematics is based on the measurements of the plane-of-sky heliocentric distances of the leading edge of CMEs, $R(t)$, expressed in units of the solar radius, $R=r / r_{\odot}{ }^{1}$. In the analysis we consider only the events

1 The $R(t)$ data utilized in the following analysis are taken from the LASCO CME Catalog compiled by Seiji Yashiro and Grzegorz Michalek under the guidance of Nat Gopalswamy: http://cdaw.gsfc.nasa.gov/CME_list/ whose leading edge position was measured in at least four instants. We employ the following CME parameters:

- $v_{\mathrm{m}}$ - mean velocity determined from the linear fit to the $R(t)$ data;

- $v_{3}$ - velocity at $R=3$ evaluated from the 2 nd degree polynomial fit to the $R(t)$ data $^{2}$;

- $a$ - mean acceleration evaluated from the 2 nd degree polynomial fit to the $R(t)$ data;

- $R_{e}$ - radial distance of the last measurements;

- $\phi$ - angular width measured at the height beyond which it remains roughly constant;

- $\psi_{\mathrm{CME}}-$ position angle ${ }^{3}$ of the CME motion.

From the original sample of soft X-ray flares we extracted 5673 events for which the positional information is available ${ }^{4}$. All of the GOES A-class flares and most of the B-class flares were lost from the original data set due to this criterion. In the analysis we employ the following flare parameters:

- $\psi_{\mathrm{F}}-$ position angle of the flare. We used the simplified expression $\tan \psi=\sin \lambda / \tan \beta$ to calculate $\psi$ from the central meridian distance $\lambda$ and the heliographic latitude $\beta$ (for details see Roša et al. 1998);

- $I_{\mathrm{p}}$ - peak flux of the X-ray flare in the GOES 1-8 A channel; - $I_{\mathrm{t}}$ - time integrated 1-8 $\AA$ flux (the fluence).

In the considered period, 1996-2001, the Solar Geophysical Data (hereafter SGD) catalogues provide positions and times for 7581 events reported as the Active prominences and filaments. From this data set we chose only the DSF-events (disappearing filaments; 1273 events). In the DSF sample only the data regarding the position and timing are $u_{\text {sed }}{ }^{5}$.

\section{Samples}

In order to associate flares to CMEs we have applied temporal and spatial criteria. First, for each CME we used the linear back-extrapolation of its trajectory to the surface of the Sun in order to estimate roughly the lift-off time $t_{0}$. Then, following the statistical procedure proposed by Dougherty et al. (2002) we estimated that the flares that occurred out of the temporal window $t_{0} \pm 0.04$ days are most likely not associated with a given CME ( 0.04 days $\approx 1 \mathrm{~h}$ ). In this way we established a set of 1321 CME-flare pairs whose relative timing was indicative of a causal relationship. Finally, we required that the position angle of the flare is within the position angle interval spanned by the CME: $\left|\psi_{\mathrm{CME}}-\psi_{\mathrm{F}}\right|<\phi / 2$. In this way we established a sample of $545 \mathrm{CME}$-flare pairs that are most likely physically related. The sample consists of 28, 153, 343 and 20 flares of the GOES intensity-class X, M, C and B, respectively. The mean angular distance from the disc center amounts to $\rho=46^{\circ} \pm 20^{\circ}$,

\footnotetext{
2 The distance $R=3$ corresponds to the average radial distance of the first measurement in the LASCO-C2 field-of-view (for details see Vršnak et al. 2004b).

${ }^{3} \psi$ is measured from the north pole in the anti-clockwise direction.

${ }^{4} \mathrm{ftp}$ ://ftp.ngdc.noaa.gov/STP/SOLAR_DATA/ SOLAR_FLARES/XRAY_FLARES/

5 ftp://ftp.ngdc.noaa.gov/STP/SOLAR_DATA/ SOLAR_FILAMENTS/
} 
which corresponds to a plane-of-sky heliocentric distance of $R=0.72$.

In order to form the sample of CMEs that were associated with the filament eruptions, i.e., to establish the sample of DSF-CMEs, an analogous procedure is applied to the leftover set of CMEs. Due to ambiguities involved in determining the actual time of disappearance of a given filament, the most appropriate time-window turned out to be larger: \pm 0.08 days $(\approx 2 \mathrm{~h})$. In this way we established a sample of $545 \mathrm{CME}-\mathrm{DSF}$ time-associated pairs. After applying the spatial criterion in the same manner as in the case of flares, we obtained a "raw" sample of 109 DSF-CMEs. Their mean angular distance from the disc center, $\rho=44^{\circ} \pm 16^{\circ}(R=0.69)$, is very similar to that of F-CMEs.

Being aware of certain inconsistencies occasionally appearing in the SGD reports of active prominences and filaments, we have checked 36 fastest DSF events whose velocities $v_{3}$ were larger than $600 \mathrm{~km} \mathrm{~s}^{-1}$, since these events are "incompatible" with the two-class CME hypothesis. Utilizing the images gained by the Extreme-Ultraviolet Imaging Telescope (EIT) aboard the Solar and Heliospheric Observatory (Delaboudiniere et al. 1995) and the Soft X-Ray Telescope (SXT, Tsuneta et al. 1991) aboard the Yohkoh satellite, we eliminated 5 out of the 36 checked events (14\%) since they were either associated with some weak flares (three cases) or with behind-the-limb flares (two cases). Nevertheless, 31 events turned to be faster than $600 \mathrm{~km} \mathrm{~s}^{-1}$ when entering the LASCO field-of-view.

So, in the following we present the data for 104 DSF-CMEs. We emphasize that in this sample there were 73 events whose source regions were not checked directly, possibly containing a certain fraction of CMEs erroneously attributed as DSF-CMEs. However, most of erroneously assigned DSF associations in the checked DSF-subset tended to be related to the fastest $\mathrm{CMEs}^{6}$, so the fraction of erroneous DSF associations in the unchecked subset is probably smaller than $14 \%$.

Finally, we also applied a more stringent spatial requirement to both samples, using $\left|\psi_{\mathrm{CME}}-\psi_{\mathrm{F}}\right|<\phi / 4$, in order to check the influence of sampling. It turned out that differences in the results are statistically insignificant. Furthermore, since the spatial criterion becomes ambiguous for large values of $\phi$, and moreover, for $\phi=360^{\circ}$ becomes meaningless, we repeated all procedures using subsamples where events with $\phi \geq 180^{\circ}$ were excluded. Although some results changed, the outcome qualitatively remained the same. In fact, the differences between F-CMEs and DSF-CMEs diminished.

Similarly, we re-inspected all distributions considering only CMEs with $\rho>45^{\circ}$ and $\rho>60^{\circ}$. Here we noticed the increase of velocities in limb events (as expected from the projection effects - see, e.g., Burkepile et al. 2004) and a slight shift of accelerations towards positive values. However, the difference between flare CMEs and DSF-CMEs remained insignificant since both samples show similar trends towards the limb.

${ }^{6}$ The five eliminated events had the "initial" velocities $v_{3}=1300$, $1110,1100,1010$, and $780 \mathrm{~km} \mathrm{~s}^{-1}$, so apparently the probability of an erroneous association decreases with the CME speed.

\section{Comparison of F-CMEs and DSF-CMEs}

\subsection{Distributions of basic CME parameters}

In Fig. 1 we compare statistical properties of the F-CMEs, DSF-CMEs and the complete CME sample. The corresponding overall statistical characteristics of the distributions (mean values, standard deviations and medians) are presented in first three columns of Table 1 .

In Fig. 1a we show the distribution of accelerations $a$. The graph reveals that there are no significant differences between accelerations of the F-CMEs and DSF-CMEs. Both samples show a large scatter of accelerations, generally ranging from -50 to $50 \mathrm{~m} \mathrm{~s}^{-2}$ and having a peak at $a \approx 0$, consistent with the distributions obtained by Moon et al. (2002). The distributions show that it is not true that the F-CMEs generally do not accelerate $(a \approx 0)$ and that the DSF-CMEs do accelerate $(a>0)$ in the LASCO C2/C3 field-of-view. For example, there is approximately the same number of $a>10 \mathrm{~m} \mathrm{~s}^{-2}$ and $a<-10 \mathrm{~m} \mathrm{~s}^{-2}$ events in both samples. However, there is a slight shift of distributions that is reflected also in the mean values (Table 1). Such a shift is qualitatively similar to that found by Moon et al. (2002), but our results show smaller differences between subsamples.

Figure $1 \mathrm{~b}$ shows the distribution of velocities at $R=3$, i.e., the velocities at the time when CMEs enter the LASCO-C2 field-of-view. The graph shows that the velocity distributions of F-CMEs and DSF-CMEs do not differ significantly. Even the asymmetries of the distributions are very similar (see the median values in Table 1). So, the velocities by which F-CMEs enter into the LASCO field-of-view are quite similar to that of the DSF-CMEs. The distributions of the mean velocities $v_{\mathrm{m}}$ are also similar for both samples (shown in the inset in Fig. 1b).

The distributions of $v_{\mathrm{m}}$ are qualitatively similar to those obtained by Moon et al. (2002) for CMEs associated with flares of SXR importance larger than C1 and non-flare CMEs. However, our median values (Table 1) are somewhat larger, and the difference between F-CMEs and DSF-CMEs is smaller. Such an outcome is due to the fact that our both samples (in DSFCMEs, as well as F-CMEs) include wider-than-average CMEs (see below), and wider CMEs are on average faster (Vršnak et al. 2004b).

Note that the mean values for DSF-CMEs that are presented in Table 1 represent a lower limit since out of the 73 unchecked events (see Sect. 3) there are probably some cases of wrong source identification - since these 73 events generally comprise slow events, the mean values in reality might be somewhat larger. For example, reducing the influence of the non-checked events by $14 \%$ would give the average value $\bar{v}_{3}=531 \mathrm{~km} \mathrm{~s}^{-1}$.

The distributions of angular widths are compared in Fig. 1c. Again one finds that the distributions of F-CMEs and DSFCMEs are quite similar. The difference of the mean values (Table 1) is primarily due to a considerably larger fraction of halo CMEs in the F-CMEs sample. If the halo CMEs are excluded, the mean widths become $91^{\circ}, 85^{\circ}$ and $61^{\circ}$ for the samples of the F-CMEs, DSF-CMEs and all CMEs.

In Fig. 1d we show the distribution of the distances up to which CMEs were traced (the last measured distance $R_{\mathrm{e}}$; 

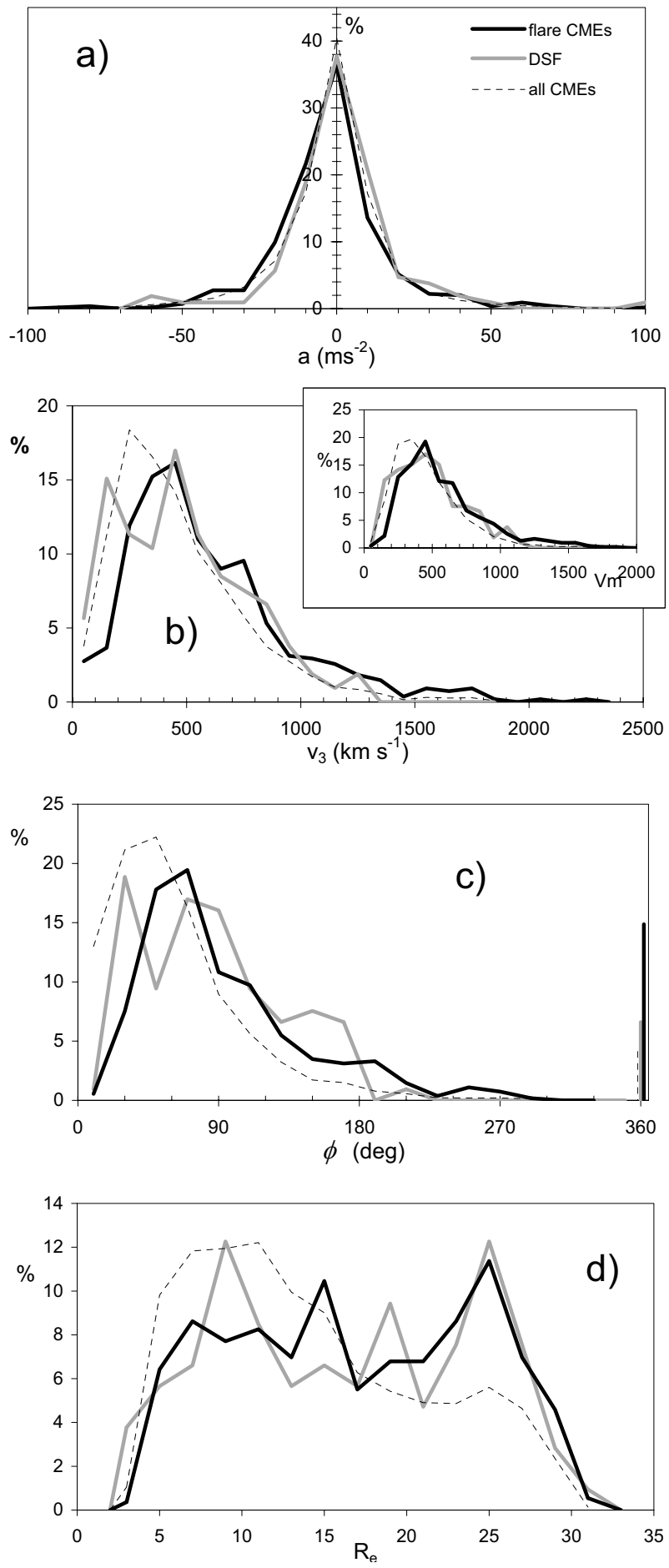

Fig. 1. Distribution of: a) accelerations ( $\Delta a=10 \mathrm{~m} \mathrm{~s}^{-2}$ bins); b) velocities at $R=3\left(\Delta v_{3}=100 \mathrm{~km} \mathrm{~s}^{-1}\right.$ bins), the inset shows the distribution of mean velocities $\left.v_{\mathrm{m}} ; \mathbf{c}\right)$ widths $\left(\Delta \phi=20^{\circ}\right.$ bins); d) radial distances of the last CME measurement ( $\Delta R=2$ bins). The sample of F-CMEs is shown by solid-black lines, the sample of DSF-CMEs by solid-gray lines and the sample of all CMEs by thin-dashed lines.

for details see Vršnak et al. 2004b). One finds that the distributions of the F-CMEs and the DSF-CMEs are very similar in this case too. Both samples show a flat distribution, i.e., a large fraction of CMEs is followed up to the edge of the LASCO-C3 field-of-view. On the other hand, the sample of all CMEs peaks at $R \approx 10$. This implies that our F-CMEs and DSF-CMEs represent a subset that comprises relatively bright CMEs remaining visible even at large distances. This is consistent with their larger-than-average widths, since wider CMEs tend to be brighter (Vršnak et al. 2004b).

\subsection{The acceleration-velocity relationship}

In Fig. 2 we show the distribution of F-CMEs and the DSF-CMEs in the acceleration-velocity space. Both samples show a similar anti-correlation of the acceleration and the velocity $a\left(v_{3}\right)$, consistent with that obtained by Vršnak et al. (2004b). A very similar outcome is also found if the velocity $v_{3}$ is replaced by $v_{\mathrm{m}}$, only the correlation is of somewhat lower statistical significance. The $a\left(v_{\mathrm{m}}\right)$ relationships based on our samples are consistent with those obtained by Moon et al. (2002), who compared different subsamples of flareCMEs with non-flare CMEs.

The pattern exposed in Fig. 2 can be explained by the aerodynamic drag (Vršnak et al. 2004b). The events faster than the solar wind generally decelerate, whereas the slower ones are still accelerating. The regression line of DSF-CMEs is somewhat steeper than that of F-CMEs, which is consistent with the smaller widths of DSF-CMEs (for details see Vršnak et al. 2004b).

It should be noted that although the overall behavior is consistent with the aerodynamic drag interpretation, there are several fast CMEs that still accelerate (see also $a\left(v_{\mathrm{m}}\right)$ graphs in Moon et al. 2002). These events can be explained by a prolonged action of the driving force during the flux rope eruption (Vršnak et al. 2004b). We emphasize that such exceptions are present in both samples and are not caused by the errors in measurement. This implies that the prolonged action of the driving force can be found in DSF-CMEs as well as in F-CMEs.

\section{The CME-flare relationship}

Although the distributions of accelerations, velocities and widths shown in Figs. 1a, b and c are quite similar for F-CMEs and DSF-CMEs, the distributions of of F-CMEs are slightly shifted to negative accelerations and higher velocities (see Table 1). This is primarily caused by the influence of large flares - in the last two columns of Table 1 we present separately the characteristics of CMEs associated with flares of the SXR importance $\mathrm{B}$ and $\mathrm{C}$, and $\mathrm{M}$ and $\mathrm{X}$. The data show that CMEs associated with more powerful flares are faster on average, and the deceleration is more prominent. A similar trend was also reported by Moon et al. (2002), however, our mean velocities are somewhat larger and our decelerations are weaker.

The differences of the mean values of $v_{3}, v_{\mathrm{m}}$ and $\phi$, between $\mathrm{M}$ and X-flare CMEs and DSF-CMEs has a statistical (t-test) significance larger than $99.99 \%$. The mean accelerations are different only at a $P=84 \%$ significance level. On the other hand, the difference between DSF-CMEs and B and C-flare CMEs is statistically insignificant $(P=43 \%, 83 \%, 54 \%$ and $73 \%$ for $a, v_{3}, v_{\mathrm{m}}$, and $\phi$, respectively). 
Table 1. Properties of the CME accelerations, velocities and widths. The number of CMEs in subsamples is denoted as $N$. The subscripts at the mean values represent the medians.

\begin{tabular}{cccccc}
\hline \hline & All CMEs & All F-CMEs & DSF-CMEs & B and C F-CMEs & M and X F-CMEs \\
& $N=4463$ & $N=545$ & $N=104$ & $N=363$ & $N=181$ \\
\hline $\bar{a} \pm \sigma_{a}\left[\mathrm{~m} \mathrm{~s}^{-2}\right]$ & $-1.2_{-0.2} \pm 20.6$ & $-1.8_{-1.6} \pm 19.7$ & $+0.1_{+1.2} \pm 16.8$ & $-1.0_{-1.0} \pm 18.2$ & $-3.4_{-3.4} \pm 22.4$ \\
$\bar{v}_{3} \pm \sigma_{v_{3}}\left[\mathrm{~km} \mathrm{~s}^{-1}\right]$ & $465_{400} \pm 295$ & $600_{506} \pm 349$ & $481_{447} \pm 285$ & $523_{460} \pm 283$ & $751_{675} \pm 414$ \\
$\bar{v}_{\mathrm{m}} \pm \sigma_{v_{\mathrm{m}}}\left[\mathrm{km} \mathrm{s}^{-1}\right]$ & $462_{408} \pm 256$ & $581_{500} \pm 314$ & $493_{475} \pm 242$ & $514_{452} \pm 255$ & $716_{635} \pm 374$ \\
$\bar{\phi} \pm \sigma_{\phi}[\mathrm{deg}]$ & $73_{53} \pm 73$ & $131_{90} \pm 107$ & $101_{85} \pm 77$ & $112_{93} \pm 76$ & $170_{116} \pm 121$ \\
\hline
\end{tabular}

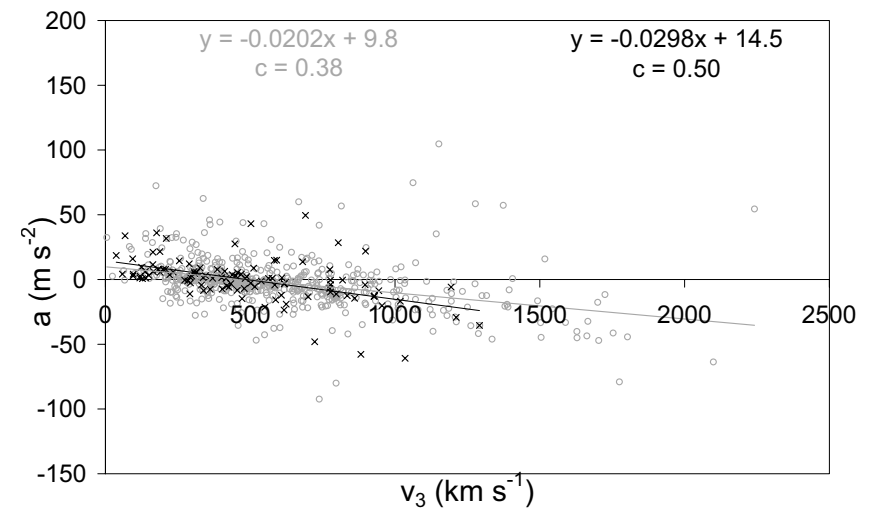

Fig. 2. The acceleration-velocity relationship, $a\left(v_{3}\right)$, of the F-CMEs (gray) and non-flare CMEs (black). The linear least squares fits (shown together with the correlation coefficient $c$ ) are almost the same for the two samples.

Such a behaviour is a consequence of the distinct relationship between the flare importance and the CME characteristics, which is illustrated by Fig. 3. In Fig. 3a we show the $v_{3}\left(I_{\mathrm{p}}\right)$ relationship for the F-CMEs (for $v_{\mathrm{m}}\left(I_{\mathrm{p}}\right)$ see Moon et al. 2003). The sample is divided into subsets according to the CME widths, and each subset is fitted by the power-law, $v_{3}=a I^{b}$. Figure 3 a reveals the distinct $v_{3}\left(I_{\mathrm{p}}\right)$ correlation. The least squares power-law fit for the complete data set reads $v_{3}=(2150 \pm 550) I_{\mathrm{p}}^{0.15 \pm 0.02}$, with the correlation coefficient $c=0.35$ and the statistical (t-test) significance larger than 99.99\%. In addition, the graph also shows that wider CMEs are systematically shifted to higher velocities.

The velocity-width relationship, $v_{3}(\phi)$, is shown in Fig. 3b separately for large flares of the SXR-classes M and X (mean importance M 6) and small flares of the classes B and C (mean importance $\mathrm{C} 4$ ). This presentation provides a direct comparison of F-CMEs and DSF-CMEs. Comparing the least squares fit lines one finds that the DSF-CMEs line (dashed) is very close to the $\mathrm{B}$ and $\mathrm{C}$ line (bold-gray), whereas the $\mathrm{M}$ and $\mathrm{X}$ subset is shifted to larger velocities (bold-black). A similar outcome would be obtained if the velocities $v_{3}$ are replaced by the CME mean velocities $v_{\mathrm{m}}$. The correlations for DSF-CMEs and $\mathrm{B}$ and $\mathrm{C}$-flare CMEs become even closer if the halo CMEs are excluded.

The relationship exposed by Figs. $3 \mathrm{a}$ and $\mathrm{b}$ indirectly implies that there is a relationship between the CME widths and the SXR importance of the associated flares. Indeed, as shown in Fig. 3c, wider CMEs tend to be associated with flares of a greater importance. The horizontal arrow drawn by the $y$-axis shows the mean width of DSF-CMEs. Again one finds that the mean widths of DSF-CMEs are very close to the mean widths of CMEs associated with the $\mathrm{B}$ and $\mathrm{C}$ class flares.

In Fig. 3d we show the dependence of the parameter $E^{*}=$ $\phi \cdot v_{\mathrm{m}}^{2}$ versus the integrated SXR flux $I_{\mathrm{t}}$. (A very similar result is obtained if $v_{\mathrm{m}}^{2}$ is replaced by $v_{3}^{2}$.) Presumably, the parameter $E^{*}$ is a proxy for the kinetic energy of a CME since a larger $\phi$ implies a larger volume, i.e., larger mass. It lacks the information on the density $\rho$, but as shown by Vršnak et al. (2004b), wider CMEs can be followed to larger heights, i.e., they are brighter, implying also denser.

What makes this dependence interesting is that it has a considerably higher correlation coefficient $(c \approx 0.5)$ than the correlations $v_{\mathrm{m}}\left(I_{\mathrm{t}}\right)$ and $\phi\left(I_{\mathrm{t}}\right)$ themselves. Since the parameter $E^{*}$ couples two parameters with lower correlation coefficients, the relatively high $c$ is even more significant.

Finally, we emphasize that we examined also the relationship between the CME acceleration and the associated SXR peak flux. We found only a very weak anti-correlation (t-test significance of 95\%) of the form $a=-(2400 \pm 1600) I_{\mathrm{p}}-$ $(1.22 \pm 0.89)$, where $a$ and $I_{\mathrm{p}}$ are expressed in $\mathrm{m} \mathrm{s}^{-2}$ and $\mathrm{W} \mathrm{m}^{-2}$, respectively. (Note that all previously mentioned relationships have a significance $>99.99 \%$.) The correlation indicates that CMEs associated with larger flares tend to show deceleration. Obviously, such a trend is a consequence of the relationship shown in Fig. 3a (stronger flare implies larger CME speed) and the tendency of fast CMEs to decelerate (Fig. 2).

\section{Counterexamples}

Bearing in mind that the LASCO-C2/C3 field-of-view covers a large range of distances, we are faced with the possibility that under certain circumstances the quadratic fit gives false values of the acceleration. For example, if a CME accelerates over some limited distance range (e.g., in the range $R<6$ which was considered by Gosling et al. 1976; and MacQueen $\&$ Fisher 1983), and then moves at a constant speed (or decelerates) untill $R=30$, its mean acceleration would be considerably underestimated. Thus, such a CME would be erroneously considered as an $a \approx 0$ event.

Consequently, there is a possibility that non-accelerating DSF-CMEs are artifacts caused by the inappropriate use of the quadratic fit. In order to eliminate such a possibility we checked the 20 fastest non-accelerating DSF-CMEs $\left(v_{3}>\right.$ $\left.750 \mathrm{~km} \mathrm{~s}^{-1} ; a \leq 0\right)$, and found that all of them really moved at an approximately constant speed (or were decelerating) in 

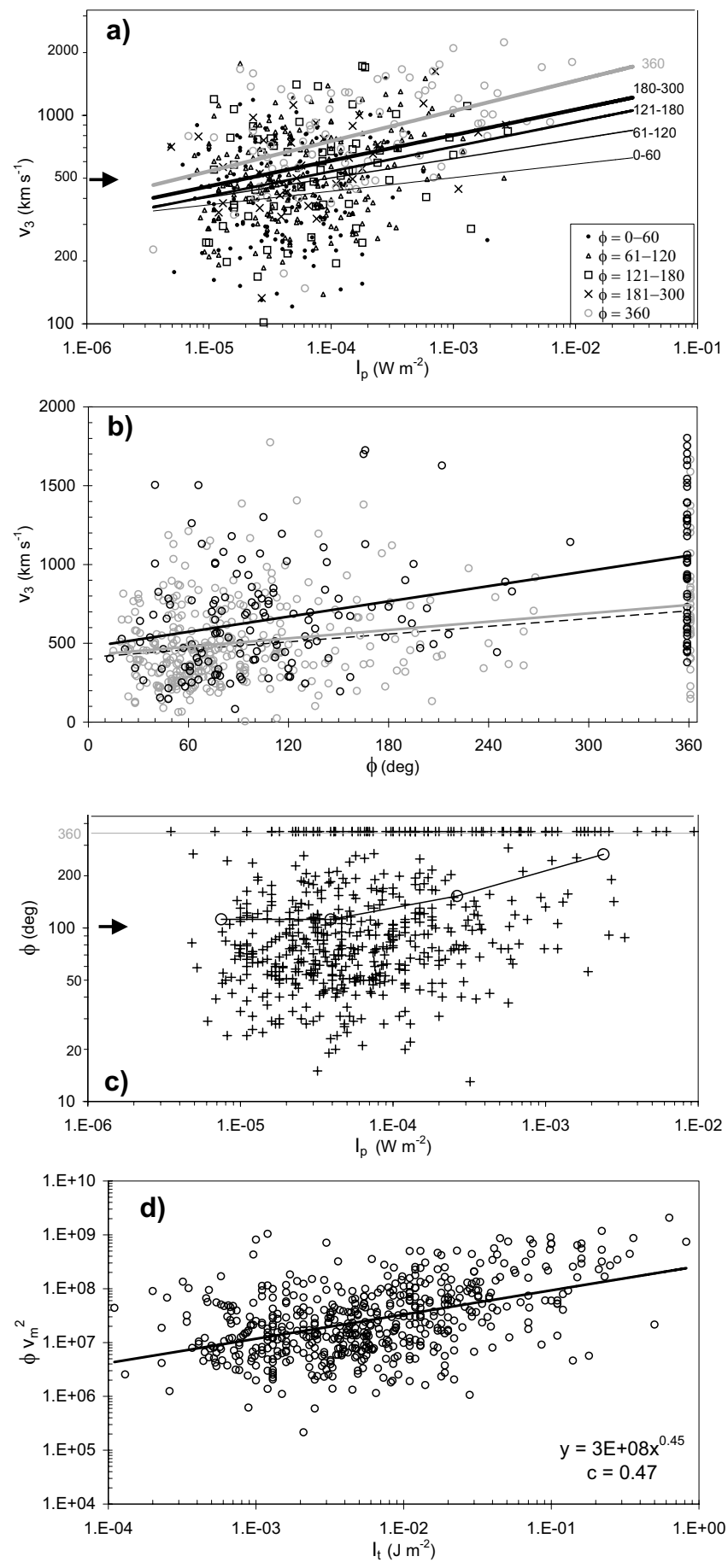

Fig. 3. a) Velocities of CMEs at $R=3$ plotted versus the SXR peak fluxes of the associated flares. The relationship is shown separately for different classes of CME widths (the bins $0-60^{\circ}, 61-120^{\circ}$, $121-180^{\circ}$, and $181-300^{\circ}$ contain $148,214,65$, and 39 events, respectively; 79 halo CMEs are shown in gray). The average velocity $\bar{v}_{3}$ of DSF-CMEs is indicated by the horizontal arrow at the $y$-axis. b) The velocity-width relationship: B and C-flare CMEs are shown in gray and $\mathrm{M}$ and $\mathrm{X}$-flare CMEs are shown in black. The fit taken from the sample of DSF-CMEs is shown by the dashed line. c) The $\mathrm{CME}$ widths shown as a function of the peak fluxes of the associated flares. The circles represent mean values of the B, C, M, and X class flares. The horizontal arrow drawn at the $y$-axis shows the mean width of DSF-CMEs. d) The parameter $E^{*}=\phi v_{\mathrm{m}}^{2}$ is shown versus the time integrated flux $I_{\mathrm{t}}$ of the associated flare. the LASCO-C2/C3 field of view. In Fig. 4a we show kinematical curves of several CMEs from this subsample. All of the presented events were associated with large quiescent filament eruptions in spotless regions, so the identification of the source region is unambiguous. The eruptions were inspected in the EIT-SOHO and SXT-Yohkoh data to confirm that they were not associated with flares (in some of events there were weak SXR enhancements recorded by GOES, but according to $\mathrm{H} \alpha$, EIT and Yohkoh images all of these weak increases were caused by remote subflares). The mean radial distance of the source region amounts to $\bar{R}=0.84 \pm 0.11$. A detailed analysis of these events will be presented in a separate paper.

The DSF-CMEs presented in Fig. 4a are clearly not compatible with the standard two-class CME concept, where such curves are expected to characterize flare-CMEs (see, e.g., kinematical curves in Andrews \& Howard 2001).

On the other hand, in Fig. 4b we show some F-CME counterexamples, selected out of $30 \mathrm{~F}-\mathrm{CMEs}$ with $a>25 \mathrm{~m} \mathrm{~s}^{-2}$ (i.e., having accelerations larger than the non-flare events considered by Andrews \& Howard 2001). The chosen events are characterized by a relatively low "initial" velocity $v_{3}$, making the effect of acceleration more prominent. We avoided events showing a complex behavior (e.g., more than one flare and/or some additional ejection in the same region), as well as events in which the predominant direction of motion departs from the position angle of the flare. The mean radial distance of the source region is $\bar{R}=0.85 \pm 0.08$, very similar to DSF-CMEs shown in Fig. 4a. Obviously, all of the presented events show characteristics that are expected for non-flare CMEs.

Note that further F-CME counterexamples can be found among the events presented in the case studies by Plunkett et al. (1997), Zhang et al. (2001), Zhang et al. (2004) and Moon et al. (2004).

\section{Discussion and conclusions}

Our results imply that the concept of two distinct classes of CMEs has to be reformulated. Figure 1 reveals that a significant fraction of F-CMEs have low velocities and there are many of them that show a considerable acceleration in the LASCO-C2 field-of-view. More specifically, around $30 \%$ of F-CMEs are slower than $400 \mathrm{~km} \mathrm{~s}^{-1}$ (i.e., slower than the slow solar wind), and around $8 \%$ have an acceleration larger than $20 \mathrm{~m} \mathrm{~s}^{-2}$ (consistent with the percentage estimated by Moon et al. 2004). A similar conclusion can be drawn from Figs. $3 \mathrm{a}$ and $3 \mathrm{~d}$ which show that weak-flare CMEs can be fast and strong-flare CMEs can be slow.

Furthermore, there are many fast DSF-CMEs showing a deceleration, i.e., not behaving as presumed in the two-class concept. Specifically, more than $30 \%$ of DSF-CMEs in our sample attained velocities higher than $600 \mathrm{~km} \mathrm{~s}^{-1}$ and more than $40 \%$ showed negative accelerations.

These statistical arguments are strengthened by various case studies (e.g., Illing \& Hundhausen 1986; Vršnak et al. 1993; Moon et al. 2004; Zhang et al. 2004; see Introduction) and by the counterexamples shown in Fig. 4.

On the other hand, it is true that CMEs associated with large flares are on average faster and larger than non-flare CMEs, 

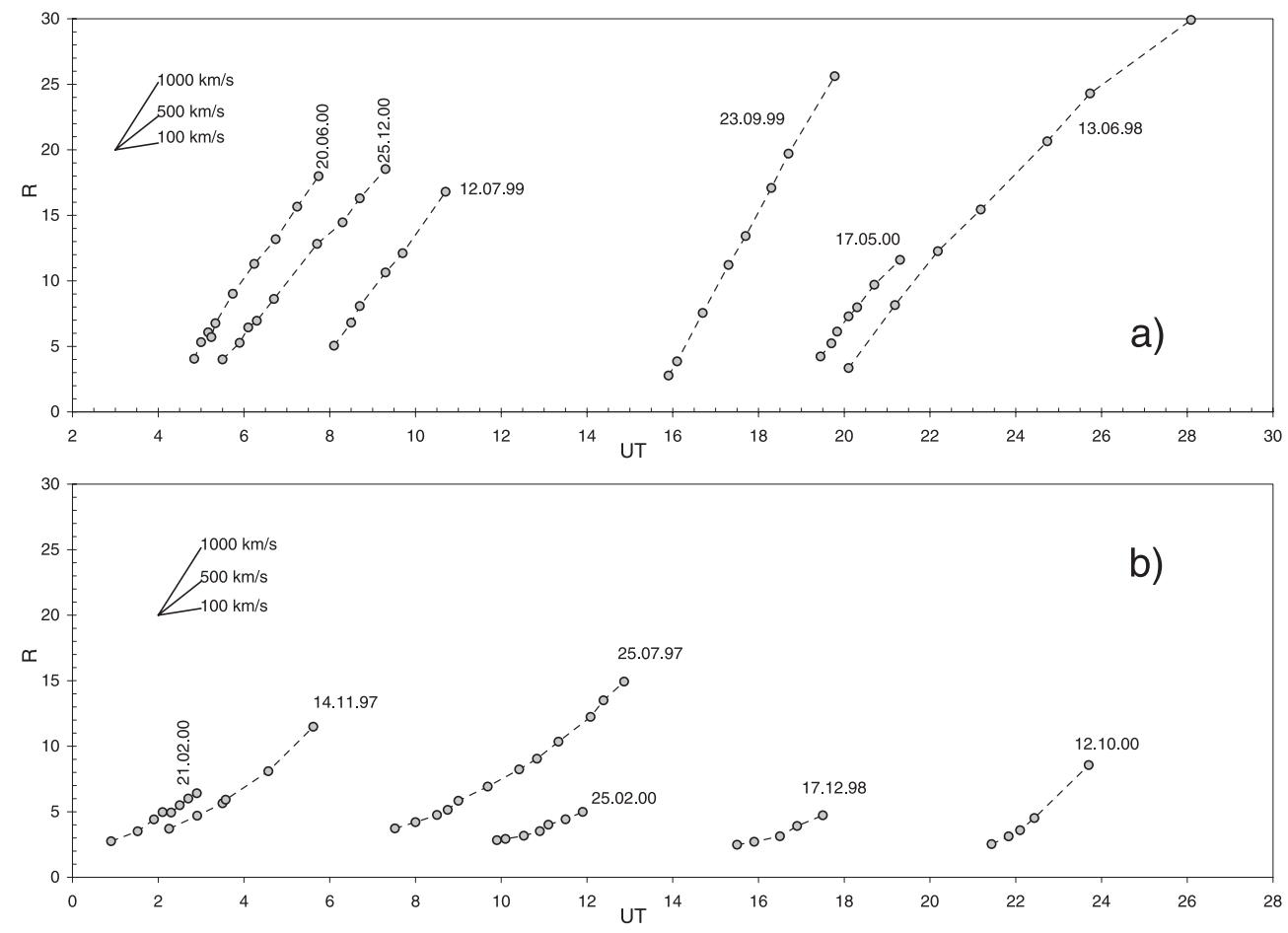

Fig. 4. a) Several examples of fast non-flare CMEs that show no acceleration, or even decelerate. b) Several examples of flare-associated CMEs showing a significant acceleration. The dates (dd.mm.yy) are attached by the curves. The slopes of 100, 500, and $1000 \mathrm{~km} \mathrm{~s}^{-1}$ are indicated in the upper-left corner of both graphs. Measurements are provided by the online LASCO CME catalogue prepared by Gopalswamy et al. (http://cdaw.gsfc.nasa.gov/CME_list/).

and that their distribution of accelerations is somewhat shifted to negative values. In fact, Figs. 3 and d show a well-defined correlation between the flare importance and the CME velocity, as well as the flare-released energy and the CME kinetic energy (see also Moon et al. 2002; Moon et al. 2003; and Burkepile et al. 2004). In these scalings, the CMEs associated with B and $\mathrm{C}$ class flares on average have characteristics very similar to the DSF-CMEs.

This implies that there is a continuum of events rather than two distinct categories, and that DSF-CMEs overlap with the "lower-energy" part of the $v\left(I_{\mathrm{p}}\right)$ scaling. Since the complete set of CMEs shows still smaller velocities and widths, one can speculate that the "weakest" events (without a readily recognizable source region) represent the lowest-speed category of CMEs. In this respect it should be emphasized that Fig. 3b indicates that the CME width represents an important parameter generally speaking, broader CMEs are faster. Consistently, the mean width of (slow) F-CMEs presented in Fig. $4 \mathrm{~b}$ amounts to only $\bar{\phi}=44^{\circ} \pm 10^{\circ}$, whereas the mean width of (fast) DSF-CMEs shown in Fig. $4 \mathrm{a}$ is $\bar{\phi}=82^{\circ} \pm 34^{\circ}$.

Such a scaling provides important information on the nature of the process of eruption. Dynamical flares (or morphologically, "two-ribbon flares"), taking place beneath CMEs, are a consequence of reconnection of the field lines stretched by the arcade eruption (e.g., Forbes 2000). The correlation of the CME velocity and the flare importance implies that the energy release in a flare is in a certain way related to the CME dynamics. However, our results indicate that the energy release itself (e.g., the heating and the resulting pressure pulse) is not directly responsible for the CME acceleration - if this would be the case, the DSF-CMEs lacking hot plasma signature, would be much slower than F-CMEs.

In this respect note that in DSF-events (especially the large ones) a growing system of post-eruption EUV loops is often observed (the loops were also observed in all of DSF-CMEs presented in Fig. 4a). Such a loop system behaves in a similar manner as the flare loop system, although it is cooler. This implies that the reconnection, analogous to that in dynamical flares, also takes place beneath the DSF-CMEs (Forbes 2000). The reason why such a reconnection does not heat the plasma to flare temperatures ${ }^{7}$ is probably related to a weaker magnetic field involved in DSF eruptions. Generally, these events are associated with the quiescent filament eruptions taking place outside active regions and are thus launched from a relatively large height (all DSF-CMEs presented in Fig. 4a are quiescent filament eruptions from spotless regions). In such an environment the plasma-to-magnetic pressure ratio $\beta$ is higher than in active regions, which directly implies that the reconnection associated with such events results in lower temperatures (Skender et al. 2003; Vršnak \& Skender 2004).

${ }^{7}$ Note that often very weak flare-like brigthenings are observed in the chromospheric or EUV spectral line images during DSF-CMEs. Sometimes such signatures are also seen in the soft X-ray images, but the associated flux increase is too weak to be recognized in the GOES (whole sun) flux measurements, especially in the periods of enhanced background flux. So, one may argue that the two-class CME concept does not make sense from this point of view too, since strictly speaking, there are no real non-flare CMEs. 
So, the results indicate that the reconnection itself influences the CME acceleration. There are two effects of reconnection process which may play an important role in the CME dynamics. Firstly, the reconnection reduces the net tension of the overlaying arcade field and increases the magnetic pressure below the erupting flux-rope (e.g., Van Tend \& Kuperus 1978; Anzer \& Pneuman 1982; Forbes 1990; Cheng et al. 2003; Lin et al. 2004). Secondly, the upward-directed reconnection jet carries the reconnected field lines to the erupting fluxrope, supplying it with a "fresh" poloidal flux. This effect enhances the "hoop-force" (e.g., Mouschovias \& Poland 1978; Chen 1989; Vršnak 1990; Chen \& Krall 2003) ${ }^{8}$, thus raising and prolonging the flux-rope acceleration (Vršnak 1990).

The feed-back effect of the reconnection is also consistent with the case studies showing that the CME acceleration is closely related to the energy release in the associated flares (e.g., Kahler et al. 1988; Zhang et al. 2001; Gallagheret al. 2003; Neupert et al. 2001; Shanmugaraju et al. 2003; Subramanian et al. 2003; Zhang et al. 2004; Vršnak et al. 2004a). The existence of the relationship between the reconnection rate and the acceleration of the ejection was presented even more directly by Wang et al. (2003), Fletcher et al. (2004) and Qiu et al. (2004), who showed that the CME acceleration is correlated with the product of the ribbon expansion-velocity and the underlying photospheric magnetic field strength. A similar conclusion can be drawn from observations presented by Zhang \& Golub (2003) who found that fast CMEs are associated with flares that show a prominent flare-ribbon expansion, whereas in flares associated with slowCMEs the expansion is less exposed (however, note that the difference is attributed therein to a different pre-eruption magnetic field structure, i.e., normal or inverse polarity configuration).

Consequently, the acceleration lasts longer in CMEs associated with gradual-flares than in the impulsive-flare CMEs, so there is a greater chance of observing the acceleration in the LASCO-C2 field-of-view (the three events denoted in Fig. 4b by boldface-labels are associated with very gradual flares), especially if the source region is close to the limb (as in the case of F-CMEs from Fig. 4b). Note that in this respect also the dimensions of eruption play a significant role (Feynman \& Ruzmaikin 2004): large features, such as quiescent filaments, starting the eruption from a relatively large height ${ }^{9}$ are more likely to show the acceleration phase in the LASCO-C2 fieldof-view, especially if their source region is close to the limb. Note that the examples presented in Fig. 4a were quiescent filament eruptions taking place close to the limb, but nevertheless finished the acceleration phase below the LASCO-C2 field-ofview.

Finally, we emphasize that our analysis concerns only the dynamics of CMEs in the 2-30 $r_{\odot}$ range. So, we do not

\footnotetext{
${ }^{8}$ Note that in the model by Chen (1989), as well as in later modificatins (e.g., Chen \& Krall 2003) the supply of the poloidal flux is attributed to the emerging flux process and not to the reconnection. On the other hand, Vršnak (1990) considered the effect of reconnection, but unfortunately, only qualitatively.

${ }^{9}$ Note that most of CMEs start to accelerate when their height is comparable to the footpoint half-separation (Vršnak et al. 1991; Chen \& Krall 2003).
}

exclude the possibility of different initiation mechanisms (see, e.g., Vršnak 2003, and references therein), nor possibly different origin of CMEs - e.g., the "ordinary" CMEs that are the consequence of the eruption of the pre-existing coronal structure, and the so-called "flare-sprays" or "ejections" (cf., Tandberg-Hanssen 1974), apparently being launched from the chromospheric layers (Bruzek \& Durrant 1977). Similarly, the analysis also does not exclude differences presumably arising due to the inverse or normal magnetic field configuration of the pre-eruption structure (e.g., Low \& Zhang 2002; Zhang \& Golub 2003).

Acknowledgements. We are grateful to the LASCO-SOHO and GOES teams for operating the instruments and performing the basic data reduction. We are especially grateful to Nat Gopalswamy, Seiji Yashiro, Grzegorz Michalek, and other colleagues who compiled the online LASCO CME Catalog. The authors thank the anonymous referee for constructive comments and suggestions.

\section{References}

Anzer, U., \& Pneuman, G. W. 1982, Sol. Phys., 79, 129

Andrews, M. D., \& Howard, R. A. 2001, Space Sci. Rev., 95, 147

Brueckner, G. E., Howard, R. A., Koomen, M. J., et al. 1995, Sol. Phys., 162, 357

Bruzek, A., \& Durrant, C. J. 1977, Illustrated Glossary for Solar and Solar-Terrestrial Physics (Dordrecht-Holland: D. Reidel), 106

Burkepile, J. T., Hundhausen, A. J., Stanger, A. L., St.Cyr, O. C., \& Seiden, J. A. 2004, J. Geophys. Res., 109

Chen, J. 1989, ApJ, 338, 453

Chen, J., \& Krall, J. 2003, J. Geophys. Res., 108, A11, 1410

Cheng, C. Z., Ren, Y., Choe, G. S., \& Moon, Y.-J. 2003, ApJ, 596, 1341

Delaboudiniere, J.-P., Artzner, G. E., Brunaud, J., et al. 1995, Sol. Phys., 162, 291

Donelly, B. R., \& Unzicker, A. 1974, NOAA Tech. Memo ELR SEL72.

Dougherty, B. L., Zirin, H., \& Hsu, K. 2002, ApJ, 577, 457

Feynman, J., \& Ruzmaikin, A. 2004, Sol. Phys., 219, 301

Fletcher, L., Pollock, J. A., \& Potts, H. E. 2004, Sol. Phys., 222, 279

Forbes, T. G. 1990, J. Geophys. Res., 95, 11919

Forbes, T. G. 2000, J. Geophys. Res., 105, 23153

Gallagher, P. T., Lawrence, G. R., \& Dennis, B. R. 2003, ApJ, 588, L53

Gosling, J. T., Hildner, E., MacQeen, R. M., et al. 1976, Sol. Phys., 48, 389

Hildner, E. 1977, in Studies of Travelling Interplanetary Phenomena, ed. M. Shea et al. (Dordrecht, Holland: D. Reidel), 3

Illing, R. M. E., \& Hundhausen, A. J. 1986, J. Geophys. Res., 91, 10951

Kahler, S. W., Moore, R. L., Kane, S. R., \& Zirin, H. 1988, ApJ, 328, 824

Lin, J., Raymond, J. C., \& van Ballegooijen, A. A. 2004, ApJ, 602, 422

Low, B. C., \& Zhang, M. 2002, ApJ, 564, L53

MacQueen, R. M., \& Fisher, R. R. 1983, Sol. Phys., 89, 89

Moon, Y.-J, Choe, G. S., Wang, H., et al. 2002, ApJ, 581, 694

Moon, Y.-J, Choe, G. S., Wang, H., Park, Y. D., \& Cheng, C. Z. 2003, J. Korean Astron. Soc., 36, 61

Moon, Y.-J, Cho, K. S., Smith, Z., et al. 2004, ApJ, 615, 1011

Mouschovias, T. Ch., \& Poland, A. I. 1978, ApJ, 220, 675

Neupert, W. M., Thompson, B. J., Gurman, J. B., \& Plunkett, S. P. 2001, JGR, 106, 25215 
Plunkett, S. P., Brueckner, G. E., Dere, K. P. 1997, Sol. Phys., 175, 699

Qiu, J., Wang, H., Cheng, C. Z., \& Gary, D. E. 2004, ApJ, 604, 900

Roša, D., Vršnak, B., Božić, H., et al. 1998, Sol. Phys., 179, 237

Shanmugaraju, A., Moon, Y.-J., Dryer, M., \& Umapathy, S. 2003, Sol. Phys., 215, 185

Sheeley, N. R. Jr., Walters, J. H., Wang, Y. -M., \& Howard, R. A. 1999, J. Geophys. Res., 104, 24739

Skender, M., Vršnak, B., \& Martinis, M. 2003, Phys. Rev. E, 68, 0464XX

St. Cyr, O. C., Burkepile, J. T., Hundhausen, A. J., \& Lecinski, A. R. 1999, J. Geophys. Res., 104, 12, 493

Subramanian, P., Ananthakrishnan, S., Janardhan, P., et al. 2003, Sol. Phys., 218, 247

Tandberg-Hanssen, E. 1974, Solar Prominences (Dordrecht-Holland: D. Reidel)

Tsuneta, S., Acton, L., Bruner, M., et al. 1991, Sol. Phys., 136, 37

Van Tend, W., \& Kuperus, M. 1978, Sol. Phys., 59, 115

Vršnak, B. 1990, Sol. Phys., 129, 295
Vršnak, B. 2003, in Energy conversion and Particle accelerationin the Solar Corona (Heidelberg: Springer), Lecture Notes Phys., 612, ed., Klein, K.-L., 28

Vršnak, B., \& Skender, M. 2004, Sol. Phys., in press

Vršnak, B., Ruždjak, V., \& Rompolt, B. 1991, Sol. Phys., 136, 151

Vršnak, B., Ruždjak, V., Rompolt, B., Roša, D., \& Zlobec, P. 1993, Sol. Phys., 146, 147

Vršnak, B., Maričić, D., Stanger, A., \& Veronig, A. 2004a, Sol. Phys., in press

Vršnak, B., Ruždjak, D., Sudar, D., \& Gopalswamy, N. 2004b, A\&A, 423, 717

Wang, H., Qiu, J., Ju, J., \& Zhang, H. 2003, ApJ, 593, 564

Yashiro, S., Gopalswamy, N., Michalek, G., et al. 2003, J. Geophys. Res., 109,

Zhang, J., Dere, K. P., Howard, R. A., Kundu, M. R., \& White S. M. 2001, ApJ, 559, 452

Zhang, J., Dere, K. P., Howard, R. A., \& Vourlidas, A. 2004, ApJ, 604, 420

Zhang, M., \& Golub, L. 2003, ApJ, 595, 1251 